\title{
Fault Sample Generation for Virtual Testability Demonstration Test Subject to Minimal Maintenance and Scheduled Replacement
}

\author{
Yong Zhang, ${ }^{1,2}$ Jing Qiu, ${ }^{1,2}$ Guanjun Liu, ${ }^{1,2}$ and Zhiao Zhao ${ }^{1,2}$ \\ ${ }^{1}$ Science and Technology on Integrated Logistics Support Laboratory, National University of Defense Technology, Changsha, \\ Hunan 410073, China \\ ${ }^{2}$ College of Mechatronic Engineering and Automation, National University of Defense Technology, Changsha, Hunan 410073, China
}

Correspondence should be addressed to Yong Zhang; zhangy21cn@126.com

Received 30 October 2014; Revised 19 January 2015; Accepted 19 January 2015

Academic Editor: Gang Li

Copyright (C) 2015 Yong Zhang et al. This is an open access article distributed under the Creative Commons Attribution License, which permits unrestricted use, distribution, and reproduction in any medium, provided the original work is properly cited.

\begin{abstract}
Virtual testability demonstration test brings new requirements to the fault sample generation. First, fault occurrence process is described by stochastic process theory. It is discussed that fault occurrence process subject to minimal repair is nonhomogeneous Poisson process (NHPP). Second, the interarrival time distribution function of the next fault event is proposed and three typical kinds of parameterized NHPP are discussed. Third, the procedure of fault sample generation is put forward with the assumptions of minimal maintenance and scheduled replacement. The fault modes and their occurrence time subject to specified conditions and time period can be obtained. Finally, an antenna driving subsystem in automatic pointing and tracking platform is taken as a case to illustrate the proposed method. Results indicate that both the size and structure of the fault samples generated by the proposed method are reasonable and effective. The proposed method can be applied to virtual testability demonstration test well.
\end{abstract}

\section{Introduction}

Recently, testability test has two basic methods, including fault injection test and field test. Both of them are physical tests. It often takes long time to get enough original fault samples in field test. In order to accelerate testability demonstration, fault injection is always applied in the testability test [1-4].

However, application results indicate that testability demonstration test based on fault injection has two unavoidable problems [1-5]. First, large numbers of fault injection tests lead to high cost. Second, some faults cannot be allowed to be injected because of destroyable influence and some faults cannot be effectively injected because of restricted fault injection means. These two shortcomings lead to unreasonable fault sample structure and low confidence.

The fault sample selection is to determine appropriate sample size and to make fault sample structure reasonable, that is, to select representative fault samples [2-6]. On one hand, considering the limits of test cost and time cost, the fault sample size needs to be as small as possible. On the other hand, in order to improve the accuracy and precision of test demonstration results, the fault sample size needs to be as large as possible. It results in a difficult contradiction [1-4].

Nowadays, many researches attach importance to virtual test. Virtual test can simulate the process of a real test and obtain test results in an efficient way. It means that virtual test can effectively decrease the test cost and risk and shorten the test period compared with physical test. According to recent studies, large-scale system modeling and simulation are difficult while small-scale system modeling and simulation can be performed in the present technical conditions [1,7-9].

As mentioned above, virtual test has many advantages, such as high efficiency, short test period, and low cost. As the fault sample size of virtual testability test is almost unlimited, it overcomes some deficiencies of physical testability test. Thus, the fault sample generation in virtual testability test 
is different from fault sample selection in physical testability test.

The combination of minimal maintenance and scheduled replacement is the main maintenance mode for many systems. The occurrences of faults are nonhomogeneous because faults occur randomly and are repairable. On the basis of Monte Carlo method, Zhao et al. proposed a fault sample generation method which was subject to exponential distribution [4]. Considering various types of life distribution and assuming perfect maintenance, Zhang et al. proposed a fault sample generation method based on renewal process [1].

The nonhomogeneous Poisson process has clear physical meanings and theoretical basis. It is widely applied to system reliability analysis, reliability indices calculation, and reliability growth test.

This paper discusses the occurrence process of faults and describes it by NHPP. A suitable fault sample simulation method for virtual testability demonstration is proposed. The main idea of the method proposed in this paper is obtaining the value and composition of fault sample based on fault statistical model and statistical simulation. The purpose is obtaining an implementation of fault occurrence within the specified time and conditions, which is called fault sample simulation in this paper.

\section{Description of Fault Occurrence Process}

Let $N(t)$ be the total number of faults up to time $t$. Faults more than two at time $t$ would be ignored under single fault assumption. So $N(t)$ has the following properties:

(1) $N(t) \geq 0$;

(2) $N(0)=0$;

(3) $N(t)$ is integer valued;

(4) the process has independent increments;

(5) $\lim _{h \rightarrow 0} P(N(t+h)-N(t)=1)=\lambda_{p}(t) h+o(h)$;

(6) $\lim _{h \rightarrow 0} P(N(t+h)-N(t) \geqslant 2)=o(h)$.

According to the definition of counting process, fault occurrence process $\{N(t), t \geq 0\}$ is a Poisson process with the parameter $\lambda_{p}(t)[10,11]$. The parameter $\lambda_{p}(t)$ is also defined as the intensity function, which describes the intensity level of fault occurrence. If $\lambda_{p}(t)$ is a constant, $\{N(t), t \geq 0\}$ is a homogeneous Poisson process (HPP). Otherwise, it is a nonhomogeneous Poisson process. The nonhomogeneous Poisson process is the generalized form of homogeneous Poisson process. Equally, the homogeneous Poisson process is the special case of a nonhomogeneous Poisson process [11].

Fault occurrence process is an independent increment process in disjoint time intervals. That is, for $t_{1}<t_{2} \leq t_{3}<$ $t_{4},\left[t_{1}, t_{2}\right)$ and $\left(t_{3}, t_{4}\right]$ are the two disjoint time intervals. In $\left[t_{1}, t_{2}\right)$ and $\left(t_{3}, t_{4}\right]$, the number of faults is $\left(N\left(t_{2}\right)-N\left(t_{1}\right)\right)$ and $N\left(t_{4}\right)-N\left(t_{3}\right)$, respectively. So $\{N(t), t \geq 0\}$ is an independent increment process. $(0, t]$;

Let $W(t)$ denote the mean number of faults in the interval

$$
W(t)=E(N(t))=\int_{0}^{t} \lambda_{p}(u) \mathrm{d} u .
$$

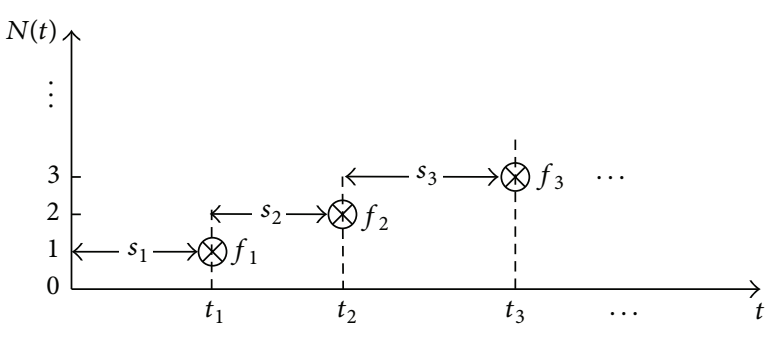

FIGURE 1: Fault occurrence process.

$W(t)$ is also called cumulative number of occurrence of failures. Thus,

$$
\lambda_{p}(t)=W^{\prime}(t)=\lim _{\Delta t \rightarrow 0} \frac{E[N(t+\Delta t)-N(t)]}{\Delta t},
$$

where $\lambda_{p}(t)$ is called the rate of occurrence of faults (ROCOF) at time $t$. It can be regarded as the mean number of faults per time unit at time $t$. If $\lambda_{p}(t)$ is a constant, $\{N(t), t \geq 0\}$ is a HPP. Otherwise, it is a NHPP. It has been verified that the mean number of faults in the interval $(0, t]$ is Poisson distributed [11].

For a nonhomogeneous Poisson process with fault occurrence intensity function $\lambda_{p}(t), N(t+s)-N(t)$ is a Poisson distribution with parameter $W(t+s)-W(t)=\int_{t}^{t+s} \lambda_{p}(u) \mathrm{d} u$, where $t \geq 0, s \geq 0 . N(t+s)-N(t)$ can be called a Poisson process with mean value $W(t+s)-W(t)$. That is,

$$
\begin{aligned}
P(N(t+s)-N(t)=n) \\
\quad=e^{-[W(t+s)-W(t)]} \cdot \frac{[W(t+s)-W(t)]^{n}}{n !} .
\end{aligned}
$$

Consider a repairable system that is put into operation at time $t=0$. The first fault event of the system will occur at time $t_{1}$. The second fault will occur at time $t_{2}$ and so on. We thus get a sequence of fault time $t_{1}, t_{2}, \ldots$. Let $s_{i}$ be the time between the $(i-1)$ th fault event and the $i$ th fault event for $i=1,2,3, \ldots$, where $s_{0}$ is taken to be zero. $s_{i}$ is called the interarrival time $i$. $\left\{s_{n}, n=1,2,3, \ldots\right\}$ is called the sequence of fault interarrival time. Fault occurrence process is indicated in Figure 1.

If fault occurrence process is a HPP having rate $\lambda_{p}$, all the fault interarrival time is independent and exponentially distributed with the same parameter $\lambda_{p}$. If the failure component is replaced or restored to an "as good as new" condition and its lifetime distribution is exponential distribution, the fault occurrence process may be a HPP. However, it is hard to meet. The rate of occurrence of faults may vary with time.

It is important to note that some fault occurrence processes do not have stationary increments. The rate of occurrence of faults varies with time rather than being a constant. This means that failures may be more or less likely to occur at certain time than others, and hence the interarrival time is generally neither independent nor identically distributed [1115].

The NHPP is generalization of HPP having the HPP as a special case. It is often used to model repairable systems that are subject to a minimal repair strategy with negligible repair 


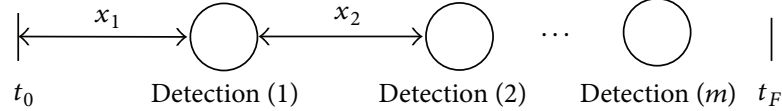

FIgURE 2: Schematic diagram of fault detection process.

time. Minimal repair means that a failed system is restored just back to functioning state. After a minimal repair, the system continues as if nothing had happened. The likelihood of a system fault is the same immediately before and after a fault.

Consider a system consisting of many components. Suppose that a component fails and causes a system failure and this component is immediately replaced by a component of the same type, thus causing a negligible system downtime. Since only a small fraction of the system is replaced, it seems natural to assume that the system's reliability after the repair essentially is the same as immediately before the failure. In other words, the assumption of minimal repair is a realistic approximation. The minimal repair assumption is therefore often applicable and the NHPP may be accepted as a realistic model [11-15].

Schematic diagram of fault detection process is shown in Figure 2. Let $x_{n}(n=1,2, \ldots)$ be the interval time of adjacent fault detection. Variables $x_{n}(n=1,2, \ldots)$ are influenced by fault occurrence process and testability plan. The number of fault detection and the interval time of fault detection are random. The number of detected faults is random, too.

Generally, the observed values of fault detection rate always change in the specified time period $(0, t]$. The formula is

$$
\gamma_{\mathrm{FD}}(t)=\frac{N_{D}(t)}{N(t)} \times 100 \%
$$

where $t$ is the time variable, $N_{D}(t)$ is the number of detected faults up to time $t$, and $N(t)$ is the total number of faults up to time $t$. As discussed above, $N_{D}(t)$ and $N(t)$ are stochastic. Thus, the observed value of fault detection rate $\gamma_{\mathrm{FD}}$ is random, too.

\section{Fault Sample Generation}

3.1. The Fault Events Simulation. Let $t_{1}, t_{2}, \ldots$ denote the successive fault event time of such a fault occurrence process. As these random variables are clearly dependent, we generate them in sequence starting with $t_{1}$ and use the generated value of $t_{1}$ to generate $t_{2}$ and so on.

Note that if a fault event occurs at time $z$, then, independent of what has occurred prior to $z$, the additional time until the next fault event has the distribution $F_{z}$. Let $\{N(z)-$ $N(z-h)=1\}$ denote that a fault event occurs at time $z(h \rightarrow 0)$. This event is recorded as $A z$. Let $t_{\Delta}$ be the interval time between $A z$ and the next fault event. Then, the event $\left\{t_{\Delta}<x\right\}$ is equal to a fault event occurring in $(z, z+x)$; that is, $\{N(z+x)-N(z)=1\}$. The interarrival time distribution function $F_{z}(x)$ of the next fault event after time $z$ is

$$
F_{z}(x)=P\left(t_{\Delta}<x \mid A z\right)=P(N(z+x)-N(z)=1 \mid A z) .
$$

According to the independent assumption of fault event, (5) can be simplified as

$$
F_{z}(x)=P(N(z+x)-N(z)=1) .
$$

According to (1) and (3), it is obtained that

$$
\begin{aligned}
F_{z}(x) & =1-P(N(z+x)-N(z)=0) \\
& =1-e^{-[W(z+x)-W(z)]} \\
& =1-e^{-\int_{z}^{z+x} \lambda_{p}(y) \mathrm{d} y}=1-e^{-\int_{0}^{x} \lambda_{p}(y+z) \mathrm{d} y} .
\end{aligned}
$$

We can now simulate the fault event time $t_{1}, t_{2}, \ldots$ by generating $t_{1}$ from the distribution $F_{0}$. Then, we generate $t_{2}$ by adding $t_{1}$ to a generated value from the distribution $F_{t_{1}}$. We generate $t_{3}$ by adding $t_{2}$ to a generated value from the distribution $F_{t_{2}}$ and so on [16].

3.2. Parametric NHPP Models of Fault Occurrence Process. The key of fault events simulation is the distribution function and its inverse function. If fault occurrence process is described by NHPP, it can be uniquely determined by the rate of occurrence of faults $\lambda_{p}(t)$. Fault occurrence processes are usually classified into linear model, power law model, and log-linear model according to the shape of the $\lambda_{p}(t)$ [11]. The three models can be expressed in the common form

$$
\lambda_{p}(t)=\lambda_{0} g(t ; \psi)
$$

where $\lambda_{0}$ is a common multiplier and $g(t ; \psi)$ determines the shape of the $\lambda_{p}(t)$.

In the linear model, the ROCOF of the NHPP is defined as

$$
\lambda_{p}(t)=\lambda_{0}(1+\alpha t) \text { for } \lambda_{0}>0, t \geq 0 .
$$

The interarrival time distribution function $F_{z}(x)$ of the next fault event after time $z$ is

$$
F_{z}(x)=1-e^{-\int_{0}^{x} \lambda_{p}(y+z) \mathrm{d} y}=1-e^{-\left(\lambda_{0} x+(1 / 2) \lambda_{0} \alpha x^{2}+\lambda_{0} \alpha z x\right)} .
$$

The inverse functionof $F_{z}(x)$ is

$$
F_{z}^{-1}(U)=-z-\frac{1}{\alpha}+\sqrt{\left(z+\frac{1}{\alpha}\right)^{2}-\frac{2}{\lambda_{0} \alpha} \ln (1-U)}
$$

for $\alpha \neq 0$.

A repairable system modeled by the linear model is deteriorating if $\alpha>0$ and improving when $\alpha<0$. When $\alpha=0$, the log-linear model reduces to a HPP. When $\alpha<$ 0 , then $\lambda_{p}(t)$ will sooner or later become less than zero. 
The model can only be used in time intervals where $\lambda_{p}(t)>0$. The linear model is often used to describe the fault occurrence process in random failure period.

In the power law model, the ROCOF of the NHPP is defined as

$$
\lambda_{p}(t)=\lambda_{0} \beta t^{\beta-1} \quad \text { for } \lambda_{0}>0, \beta>0, t \geq 0 .
$$

Thus,

$$
\begin{gathered}
F_{z}(x)=1-e^{-\int_{0}^{x} \lambda_{p}(y+z) \mathrm{d} y}=1-e^{\lambda_{0} t^{\beta}-\lambda_{0}(x+z)^{\beta}}, \\
F_{z}^{-1}(U)=-z+\sqrt[\beta]{z^{\beta}-\frac{\ln (1-U)}{\lambda_{0}}} .
\end{gathered}
$$

A repairable system modeled by the power law model is seen to be improving if $0<\beta<1$, and deteriorating if $\beta>1$. If $\beta=0$, the model reduces to a HPP. This NHPP is sometimes referred to as a Weibull process, since the ROCOF has the same functional form as the failure rate function of the Weibull distribution. The power law model is often used to describe the fault occurrence process of electromechanical systems and reliability growth model.

In the log-linear model, the ROCOF of the NHPP is defined by

$$
\lambda_{p}(t)=\lambda_{0} e^{\beta t} \quad \text { for } \lambda_{0}>0, t \geq 0
$$

Thus,

$$
\begin{gathered}
F_{z}(x)=1-e^{-\int_{0}^{x} \lambda_{p}(y+z) \mathrm{d} y}=1-e^{-\left(\lambda_{0} / \beta\right)\left[e^{\beta(x+z)}-e^{\beta z}\right]}, \\
F_{z}^{-1}(U)=\frac{\ln \left[e^{\beta z}-(\beta / \lambda) \ln (1-U)\right]}{\beta}-z \text { for } \beta \neq 0 .
\end{gathered}
$$

A repairable system modeled by the log-linear model is improving if $\beta<0$ and deteriorating if $\beta>0$. When $\beta=0$, the log-linear model reduces to a HPP. The log-linear model is often used to describe the fault occurrence process of electronic systems.

The cumulative number of faults and occurrence time of each fault subject to specified conditions and time period can be obtained according to reliability test and other trials. Appropriate parametric NHPP model will be selected according to failure statistics. We do not intend to discuss the model selection and parameter estimation method in this paper.

3.3. Fault Sample Simulation. In this paper, we assume that the repair or maintenance time is negligible and the corrective maintenance is minimal maintenance or repair, that is, the maintenance action which restores the part to the failure rate it had when it failed. The part after repair is as bad as old.

If fault events occur before the scheduled replacement, the part will be processed by breakdown maintenance. If no fault event occurs before scheduled replacement, the part should be replaced by a brand new one regardless of its health condition when it meets the replacement requirement.

Statistical simulation method is also known as random simulation method, random sampling method, or statistical test method. It can effectively solve uncertainty problems and complex computing problems. For example, Monte Carlo method is widely applied in financial engineering, statistical physics, computational mathematics, reliability engineering, and other fields $[17,18]$.

The flow chart of fault sample simulation is showed in Figure 3. $T_{w}$ is the scheduled interval replacement time. $F_{z}(x)$ is the interarrival time distribution function of the next fault event after time $z \cdot u_{i}(i=1,2, \ldots)$ are uniform $(0,1)$ random variables. $s_{i}(i=1,2, \ldots)$ are the simulation results of interarrival time of fault events. $S[p]$ is the cumulative working time of the $p$ th part. The initial value of $S[p]$ is zero for $p=1,2,3, \ldots j$ is the cumulative number of fault events. $t_{j}$ is the occurrence time of the $j$ th fault event. ST is the cumulative working time of the parts. $T^{*}$ is the specified statistical time.

The basic steps of fault sample simulation are as follows.

Step 1. Determine the parameters of the NHPP and set the interval replacement time $T_{w}$.

Step 2. Initialize the specified statistical time $T^{*}$ and initialize $z=0, i=0, j=0, t_{j}=0, p=1, S[p]=0$, and $S T=0$.

Step 3. Solve the interarrival time distribution function $F_{z}(x)$ of the next fault event after time $z$.

Step 4. Solve the inverse function $F_{z}^{-1}(U)$ of $F_{z}(x)$.

Step 5. Generate the random number $u_{i}$.

Step 6. Calculate the interarrival time $s_{i}$ based on the direct sampling method, $s_{i}=F_{z}^{-1}\left(u_{i}\right)$.

Step 7. If $S[p]+s_{i}<T_{w}$, it shows that the part has broken down before the scheduled replacement; the cumulative working time of this part is $S[p]=S[p]+s_{i}$. The cumulative number of fault events adds 1 ; that is, $j=j+1$. Then, set $t_{j}=S[p], z=S[p]$, and $S T=S T+S[p]$. If $S[p]+s_{i} \geq T_{w}$, it shows that the part is good until the scheduled replacement time. The part is as good as new after replacement. Then, set $S[p]=S[p]+T_{w}, z=0, S T=S T+S[p]$, and $p=p+1$.

Step 8. If $S T \geq T^{*}$, stop.

Step 9. Obtain fault samples based on probability proportional to size (PPS) sampling method. Each fault mode is set to be proportional to its occurrence percentage ratio (OPR).

\section{Examples}

An automatic pointing and tracking platform has the ability to isolate the movement of moving vehicles, such as car, ship, and aircraft. It can automatically track the target and maintain stable communication. The stable tracking platform consists of multiple subsystems. We take antenna driving subsystem as example to carry out experiments. The lifetime of the automatic pointing and tracking platform is 15 years. The average working time is 1500 hours per year. The lifetime 


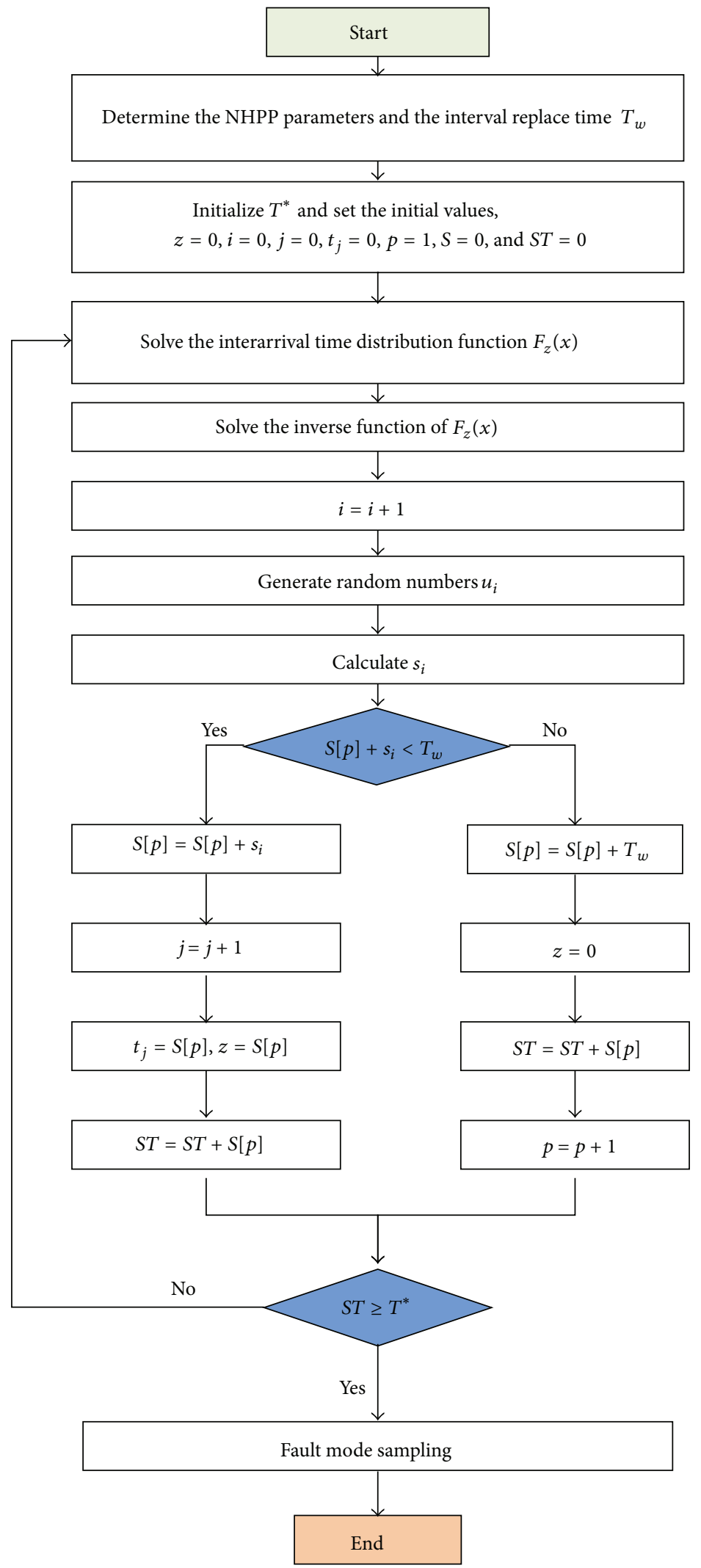

FIGURE 3: The flow chart of fault sample simulation. 
TABLE 1: Fault modes and their occurrence percentage ratio.

\begin{tabular}{|c|c|c|c|}
\hline Code & Fault mode & Fault unit & OPR \\
\hline $\mathrm{A} 1$ & No signal output & Antenna & $7.1 \%$ \\
\hline $\mathrm{A} 2$ & Unstable signal output & Antenna & $3.7 \%$ \\
\hline A3 & No rotation output & Pitching motor & $10.7 \%$ \\
\hline A4 & Angle output tolerance & Pitching motor & $7.1 \%$ \\
\hline A5 & No turning signal output & Pitching motor driver & $12.5 \%$ \\
\hline A6 & $\begin{array}{l}\text { Turning signal output } \\
\text { tolerance }\end{array}$ & Pitching motor driver & $5.4 \%$ \\
\hline A7 & No rotation output & Azimuth motor & $8.9 \%$ \\
\hline A8 & Angle output tolerance & Azimuth motor & $5.4 \%$ \\
\hline A9 & No turning signal output & Azimuth motor driver & $10.7 \%$ \\
\hline $\mathrm{A} 10$ & $\begin{array}{l}\text { Turning signal output } \\
\text { tolerance }\end{array}$ & Azimuth motor driver & $7.1 \%$ \\
\hline A11 & No control signal output & Motion control card & $8.9 \%$ \\
\hline A12 & $\begin{array}{l}\text { Control signal output } \\
\text { error }\end{array}$ & Motion control card & $12.5 \%$ \\
\hline
\end{tabular}

of the antenna drive subsystem is 7500 working hours. The antenna driving subsystems are replaced by new ones every 5 years. In the subsystem's life cycle, breakdown maintenance and the assumption of minimal repair with negligible repair times are taken when it fails. The subsystems are replaced by new ones with the assumption of perfect repair after the end of their life cycle.

As the platform is new equipment, the failure statistics in full life cycle are poor. The same antenna driving subsystems have been tried out for 5 years in advance. We collected some credible and valuable failure and maintenance statistics of the subsystem in their single life cycle. The statistics contain 56 complete sets of trial data. Fault modes and their occurrence percentage ratio of the antenna driving subsystem are shown in Table 1. The fault occurrence process of the antenna driving subsystem in its single life cycle is a NHPP. The parameters of ROCOF were estimated by maximum likelihood method [11]. We obtained a NHPP in $\left(0, T^{*}\right)$ and faults occurred at time $t_{1}, t_{2}, \ldots$..

$\widehat{\beta}$ can be found by solving

$$
\sum_{i=1}^{n} t_{i}+\frac{n}{\beta}-\frac{n T^{*}}{1-e^{-\beta T^{*}}}=0 .
$$

Then, solve $\hat{\lambda}_{0}$ by

$$
\widehat{\lambda}_{0}=\frac{n \widehat{\beta}}{e^{\widehat{\beta} T^{*}}-1} .
$$

The result is $\lambda_{p}(t)=0.00054 e^{0.00022 t}$.

The interarrival time distribution function $F_{z}(x)$ of the next fault event after time $z$ of the antenna driving subsystem is

$$
\begin{aligned}
F_{z}(x) & =1-e^{-\int_{0}^{x} 0.00054 e^{0.00022(y+z)} \mathrm{d} y} \\
& =1-e^{(27 / 11)\left[e^{0.00022 z}-e^{0.00022(x+z)}\right]} .
\end{aligned}
$$

\begin{tabular}{|c|c|c|}
\hline Number & Working time (h) & Fault \\
\hline 1 & 1287 & $\mathrm{~A} 12$ \\
\hline 2 & 4422 & A10 \\
\hline 3 & 5953 & A3 \\
\hline 4 & 6900 & A11 \\
\hline 5 & 7166 & A5 \\
\hline 6 & 7261 & $\mathrm{~A} 2$ \\
\hline 7 & 7482 & $\mathrm{~A} 1$ \\
\hline 8 & 9833 & A7 \\
\hline 9 & 10061 & A11 \\
\hline 10 & 10963 & $\mathrm{~A} 3$ \\
\hline 11 & 12487 & A9 \\
\hline 12 & 13369 & $\mathrm{~A} 1$ \\
\hline 13 & 13932 & A5 \\
\hline 14 & 14392 & A12 \\
\hline 15 & 14554 & $\mathrm{~A} 10$ \\
\hline 16 & 14740 & A4 \\
\hline 17 & 14815 & A9 \\
\hline 18 & 14870 & $\mathrm{~A} 12$ \\
\hline 19 & 16008 & A6 \\
\hline 20 & 17212 & $\mathrm{~A} 3$ \\
\hline 21 & 18181 & $\mathrm{~A} 10$ \\
\hline 22 & 19384 & A6 \\
\hline 23 & 21639 & A11 \\
\hline 24 & 21784 & A5 \\
\hline 25 & 21903 & A7 \\
\hline 26 & 22032 & A12 \\
\hline 27 & 22208 & A5 \\
\hline 28 & 22316 & A8 \\
\hline 29 & 22354 & A4 \\
\hline
\end{tabular}

TABLE 2: A simulation result of fault sample.

The inverse function of $F_{z}(x)$ is

$$
F_{z}^{-1}(U)=\frac{50000}{11} \ln \left[e^{0.00022 t}-\frac{11}{27} \ln (1-U)\right]-z
$$

It is assumed that the specified statistical time of testability demonstration is 15 years. The fault samples are generated by fault occurrence process simulation based on the proposed method. The fault modes and their occurrence time are obtained. A simulation result of fault sample is shown in Table 2.

The cumulative number of the subsystem faults is shown in Figure 4. Abscissa represents the cumulative working time. Ordinate represents the cumulative number of faults. The cumulative number of faults increases one when a fault occurs.

We implement the statistical simulation 1000 times. The specified statistical simulation time is single life cycle of the antenna driving subsystem. 1000 groups of fault samples are generated automatically by simulation. The numbers of faults in fault sample are random variables. We compare some statistics of the actual samples and the simulation samples to examine the effectiveness of the proposed method. The 


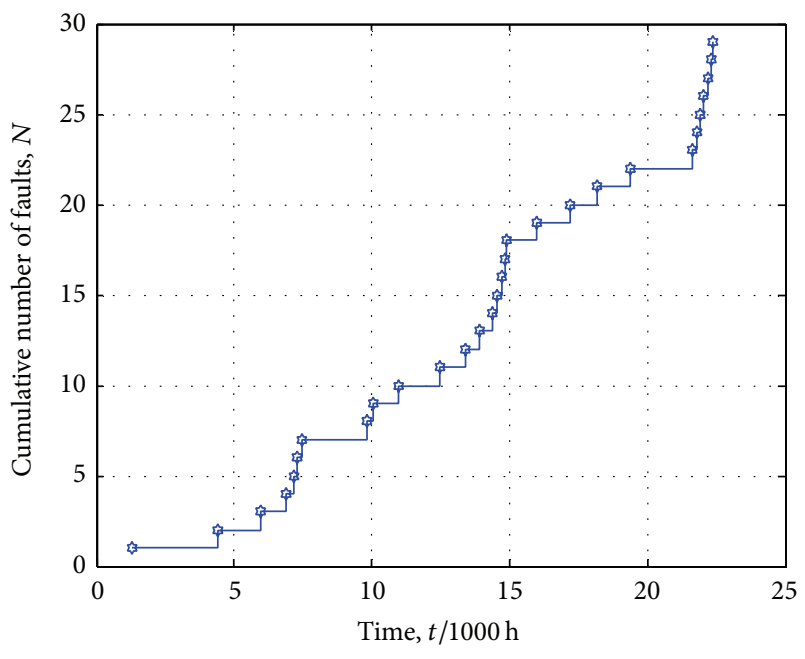

FIGURE 4: Cumulative number of faults.

TABLE 3: The comparison of sample statistics.

\begin{tabular}{lcc}
\hline Statistic & $\begin{array}{c}\text { The actual } \\
\text { samples }\end{array}$ & $\begin{array}{c}\text { The simulation } \\
\text { samples }\end{array}$ \\
\hline Sample mean & 10.46 & 10.57 \\
Sample variance & 2.35 & 2.29 \\
Two-order origin moment & 118.26 & 121.64 \\
Sample median & 10.5 & 11 \\
Percentage ratio of A1 & $7.1 \%$ & $6.9 \%$ \\
Percentage ratio of A2 & $3.7 \%$ & $4.0 \%$ \\
Percentage ratio of A3 & $10.7 \%$ & $10.5 \%$ \\
Percentage ratio of A4 & $7.1 \%$ & $7.1 \%$ \\
Percentage ratio of A5 & $12.5 \%$ & $12.4 \%$ \\
Percentage ratio of A6 & $5.4 \%$ & $5.6 \%$ \\
Percentage ratio of A7 & $8.9 \%$ & $8.7 \%$ \\
Percentage ratio of A8 & $5.4 \%$ & $5.6 \%$ \\
Percentage ratio of A9 & $10.7 \%$ & $10.5 \%$ \\
Percentage ratio of A10 & $7.1 \%$ & $7.5 \%$ \\
Percentage ratio of A11 & $8.9 \%$ & $8.8 \%$ \\
Percentage ratio of A12 & $12.5 \%$ & $12.4 \%$ \\
\hline
\end{tabular}

comparison is shown in Table 3. Let $m_{i}$ denote the number of faults of the $i$ th fault sample. The sample mean is

$$
\bar{m}=\sum_{i=1}^{k} m_{i}
$$

where $k$ is the sample size.

The sample variance is

$$
v^{2}=\frac{1}{k-1} \sum_{i=1}^{k}\left(m_{i}-\bar{m}\right)^{2} .
$$

The two-order origin moment is

$$
A_{2}=\frac{1}{k} \sum_{i=1}^{k} m_{i}^{2} .
$$

The sample values are arranged in increasing order so as to meet $m_{1} \leq m_{2} \leq \cdots \leq m_{k-1} \leq m_{k}$.

The sample median is

$$
\widetilde{m}= \begin{cases}m_{(k+1) / 2} & k \text { is an odd number } \\ \frac{1}{2}\left(m_{k / 2}+m_{(k / 2)+1}\right) & k \text { is an even number. }\end{cases}
$$

The percentage ratios of fault modes are also figured out and compared in Table 3.

We can get that the statistics of the simulation results are nearly consistent with the actual fault samples according to the comparison. The composition of the simulation samples is rational. The results show that the proposed method is feasible and effective. The random fault samples generated by statistical simulation can be applied to virtual testability demonstration test.

\section{Conclusion}

(1) It is analyzed and pointed out that the fault sample generation in virtual testability test is different from fault sample selection in physical testability test.

(2) In the case of minimal repair and scheduled replacement, the fault occurrence process can be described by NHPP theory. A fault sample generation approach for virtual testability demonstration test is proposed.

(3) As some assumptions are eliminated, the size and structure of the fault samples simulated by proposed method are reasonable. Experiment results show that the proposed method is feasible and effective. It can also be applied to virtual maintainability test and integrated logistics support scheme design.

\section{Notations}

$\begin{array}{ll}N(t): & \begin{array}{l}\text { The number of detected faults up to } \\ \text { time } t\end{array} \\ N_{D}(t): & \begin{array}{l}\text { The number of detected faults up to } \\ \text { time } t\end{array} \\ \gamma_{\mathrm{FD}}: & \text { Fault detection rate } \\ n: & \text { Fault sample size } \\ W(t): & \begin{array}{l}\text { Mean number of faults in the interval } \\ (0, t]\end{array} \\ \lambda_{p}(t): & \begin{array}{l}\text { The rate of occurrence of faults at time } t \\ x_{n}(n=1,2, \ldots):\end{array} \\ A z: & \begin{array}{l}\text { The interval time of adjacent fault } \\ \text { detection }\end{array} \\ t_{\Delta}: & \begin{array}{l}\text { The event denoting one occurring fault } \\ \text { The interval time between } A z \text { and the }\end{array} \\ F_{z}(x): & \begin{array}{l}\text { next fault event } \\ \text { Interval time distribution function of } \\ \text { the next fault event after time } z\end{array} \\ g(t ; \psi): & \begin{array}{l}\text { The coefficient determines the shape of } \\ \text { the } \lambda_{p}(t)\end{array} \\ T_{w}: & \begin{array}{l}\text { The scheduled interval replacement } \\ \text { time }\end{array} \\ u_{i}(i=1,2, \ldots): & \begin{array}{l}\text { Random variables having the uniform } \\ \text { distribution in }(0,1)\end{array}\end{array}$


$s_{i}(i=1,2, \ldots)$ : The time $t$ between the $(i-1)$ th fault event and the $i$ th fault event

$S[p]: \quad \quad$ The cumulative working time of the $p$ th part

$j: \quad$ The cumulative number of fault events

$t_{j}: \quad$ The occurrence time of the $j$ th fault event

ST: $\quad$ The cumulative working time of the parts

$T^{*}$ : The specified statistical time.

\section{Conflict of Interests}

The authors declare that there is no conflict of interests regarding the publication of this paper.

\section{Acknowledgments}

This research was supported by Research Fund for Shanghai Astronautics Science and Technology and National Natural Science Foundation of China. The authors would like to thank Peng Yang, Kehong Lv, and Chenxu Zhao for enlightening discussions. They would also like to thank Chao Wang and Chao Wu for correction.

\section{References}

[1] Y. Zhang, J. Qiu, G. Liu, and P. Yang, "A fault sample simulation approach for virtual testability demonstration test," Chinese Journal of Aeronautics, vol. 25, no. 4, pp. 598-604, 2012.

[2] T. M. Li, Research on optimization design and integrated evaluation of testability verification test for equipments [Ph.D. thesis], National University of Defense Technology, Changsha, China, 2010.

[3] L. Liu, J. Y. Chang, and M. G. Zhou, "Selection and simulation of fault samples in weapon equipment's maintainability test," in Proceedings of the 8th International Symposium on Test Measure, Chongqing, China, August 2009.

[4] C. X. Zhao, G. J. Liu, J. Qiu, and Y. Zhang, "Fault samples simulation based on Monte Carlo method in testability virtual test," in Proceedings of the 9th International Conference on Reliability, Maintainability and Safety (ICRMS '11), pp. 358-362, June 2011.

[5] T. Li, J. Qiu, and G. Liu, "New methodology for determining testability integrated test scheme with test data in the development stages," Journal of Mechanical Engineering, vol. 45, no. 8, pp. 52-57, 2009.

[6] H. X. Wang, S. S. Liu, and X. H. Ye, "Study on the method for fault sample selection based on fuzzy clustering," in Proceedings of the International Conference on Information Science, Automation and Material System, pp. 21-22, 2011.

[7] Z. Q. Jia and J. Y. Cai, "Performance reliability estimation of small sample in multi-testing environments based on circuit simulation and hypothesis of normal distribution," Acta Aeronautica et Astronautica Sinica, vol. 31, no. 4, pp. 791-796, 2010.

[8] A. Abdul Kadir, X. Xu, and E. Hämmerle, "Virtual machine tools and virtual machining-a technological review," Robotics and Computer-Integrated Manufacturing, vol. 27, no. 3, pp. 494508, 2011.

[9] Z. X. Li, X. P. Yan, C. Q. Yuan, Z. Peng, and L. Li, "Virtual prototype and experimental research on gear multi-fault diagnosis using wavelet-autoregressive model and principal component analysis method," Mechanical Systems and Signal Processing, vol. 25, no. 7, pp. 2589-2607, 2011.

[10] S. P. Tan, H. T. Yuan, and L. L. Han, "Analysis of fault stochastic process for armored vehicles," Acta Armamentarii, vol. 27, no. 6, pp. 961-964, 2006.

[11] M. Rausand, System Reliability Theory: Models, Statistical Methods and Applications, Wiley, New York, NY, USA, 2003.

[12] J. L. Coetzee, "The role of NHPP models in the practical analysis of maintenance failure data," Reliability Engineering \& System Safety, vol. 56, no. 2, pp. 161-168, 1997.

[13] V. V. Krivtsov, "Practical extensions to NHPP application in repairable system reliability analysis," Reliability Engineering and System Safety, vol. 92, no. 5, pp. 560-562, 2007.

[14] C. L. Ao, Y. J. Li, X. B. Yan, and F. F. Chu, "Operational reliability of tractor engines based on non-homogeneous poisson process," Chinese Journal of Mechanical Engineering, vol. 43, no. 10, pp. 206-210, 2007.

[15] Z. Ming, J. Tao, Y. Zhang, X. Yi, and X. Chen, "Bayesian reliability-growth analysis for statistical of diverse population based on non-homogeneous poisson process," Chinese Journal of Mechanical Engineering, vol. 22, no. 4, pp. 535-541, 2009.

[16] S. M. Ross, Simulation, Elsevier Press, Amsterdam, The Netherlands, 2006.

[17] J. P. Yao, C. Tan, and L. Zhou, "Research on assembly quality evaluation for aircraft product based on Monte-Carlo simulation," Advanced Science Letters, vol. 4, no. 6-7, pp. 2352-2355, 2011.

[18] M. S. Singh, R. K. B. Singh, R. Khatri, and B. I. Sharma, "Monte Carlo simulation study of electron beam interaction in multilayered semiconducting materials," Advanced Science Letters, vol. 3, no. 1, pp. 57-61, 2010. 


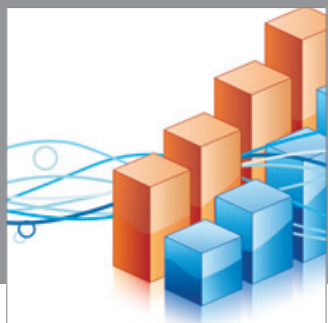

Advances in

Operations Research

mansans

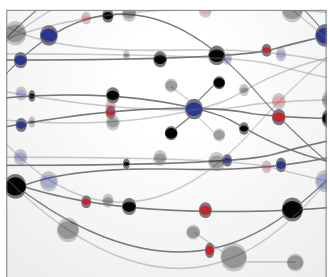

The Scientific World Journal
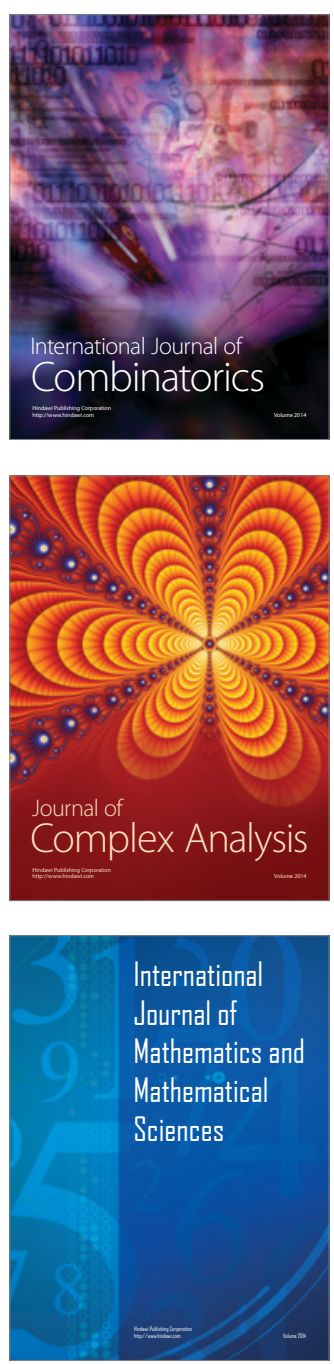
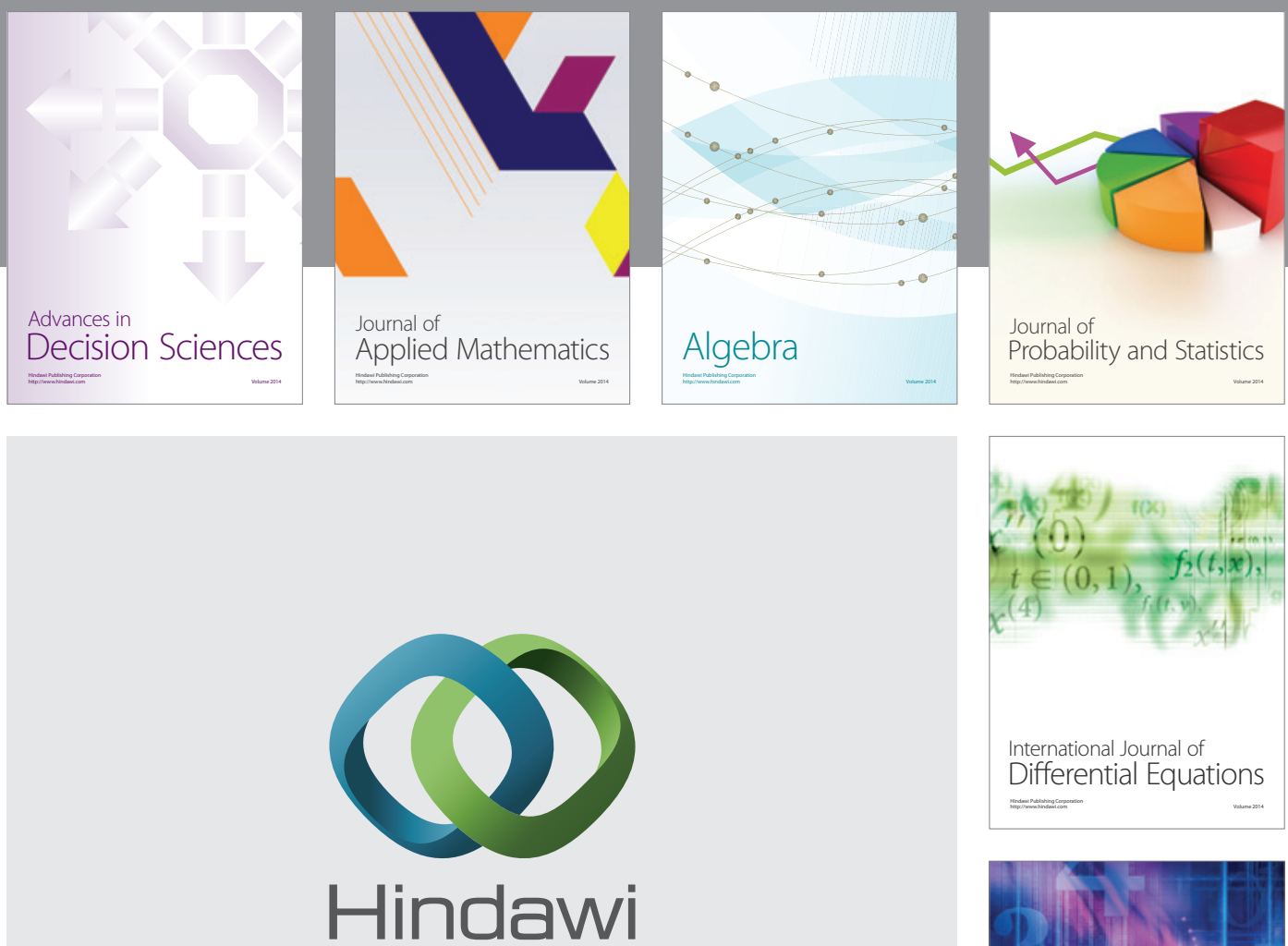

Submit your manuscripts at http://www.hindawi.com
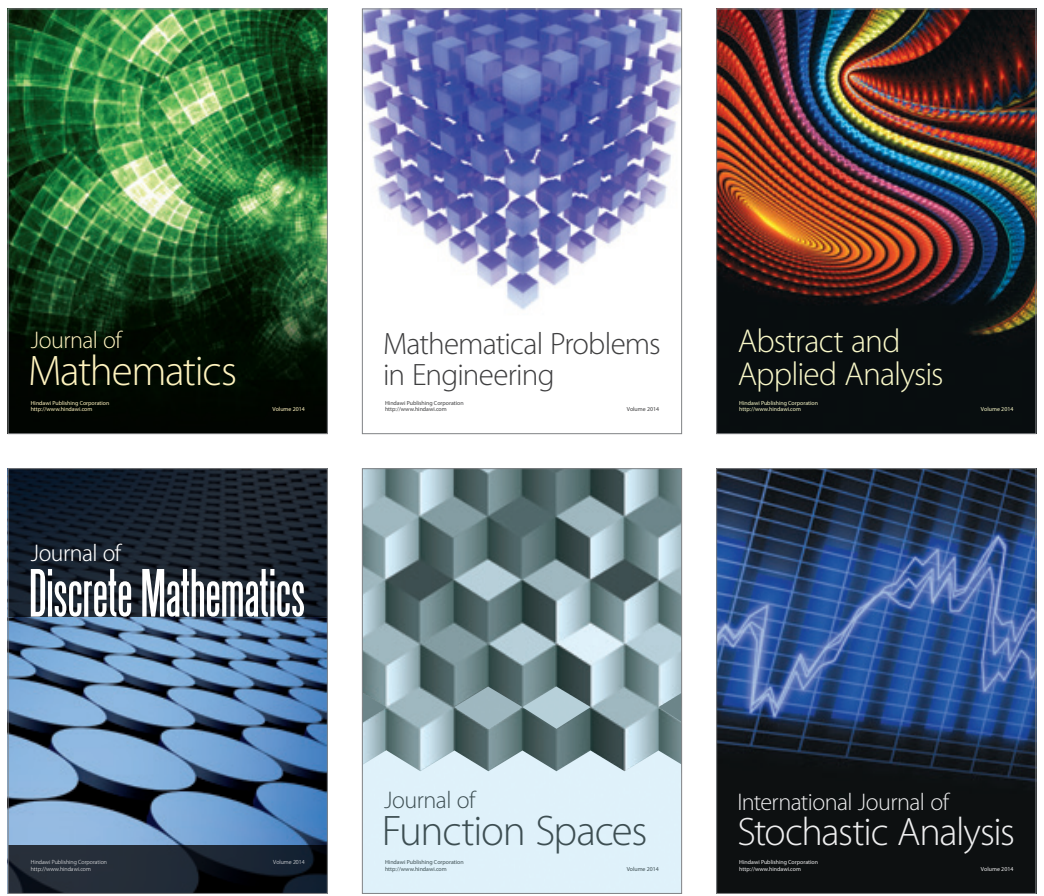

Journal of

Function Spaces

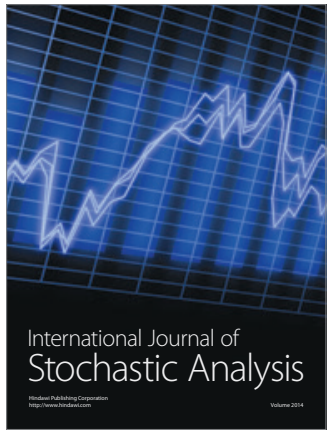

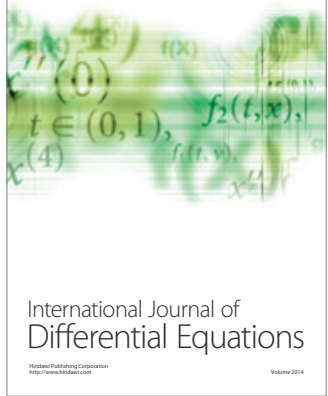
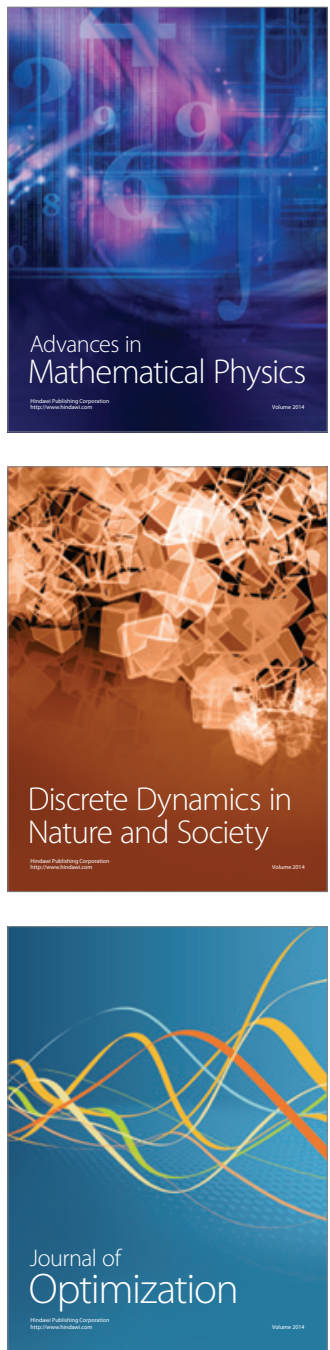